\title{
A INSERÇÃO DO NUTRICIONISTA NA ESTRATÉGIA DA SAÚDE DA FAMÍLIA: O OLHAR DE DIFERENTES TRABALHADORES DA SAÚDE
}

\author{
THE INSERTION OF THE NUTRITIONIST ON FAMILY HEALTH PROGRAM:
} A DIFFERENT VIEWS OF HEALTH WORKERS

\section{INSERCIÓN DEL NUTRICIONISTA EN LA ESTRATEGIA DE SALUD DE LA FAMILIA: LA POSICIÓN DE LOS DEMÁS PROFESIONALES DE SALUD}

Anderson Carlos dos Santos*

* Nutricionista. Especialização em Saúde da Família, Perceptor na Residência Multiprofissional em Saúde da Família - UFPR.

\begin{abstract}
RESUMO. Este estudo buscou discutir a atuação e importância do nutricionista na Estratégia de Saúde da Família, sob o olhar de diferentes trabalhadores de saúde, que desempenham suas funções em Unidades Básicas de Saúde (UBS) e também nas Unidades de Saúde da Família (UBSF) do Município de Colombo, PR. Trata-se de estudo exploratório, cujo principal objetivo foi avaliar o conhecimento destes profissionais, sobre o papel do nutricionista em programas de Saúde da Família. Os dados foram obtidos em entrevistas semi-estruturadas realizadas com 12 profissionais de saúde. Seguiu-se o preconizado na resolução 196/96, que regulamenta a pesquisa com seres humanos. Dos dados obtidos foi possível construir as seguintes categorias: CONHECENDO O NUTRICIONISTA e O NUTRICIONISTA PARTICIPANDO DO PSF. Os resultados obtidos demonstram que os trabalhadores de Saúde, das mais diversas áreas de atuação, sentem a necessidade de que o profissional nutricionista se insira nas equipes de saúde e reivindicam que o campo de atuação deste profissional seja ampliado, e que o nutricionista, possa somar seus conhecimentos com os demais membros das equipes de saúde e, principalmente os repasse à população, esta deve ser o centro de toda a atenção à saúde.
\end{abstract}

PALAVRAS-CHAVE: nutricionista; programa saúde da família; trabalho.

ABSTRACT. This study discuss the action and importance of the nutritionist on Family Health Program from different views of health workers at Basic Health Unit in Colombo city - Brazil. It is an exploratory study with the objective to evaluate the knowledge of the workers about the nutritionist's roles on Family Health Program (FHP). The data were collected through interview with 12 health professionals. It permits to construct the following categories: KNOWING THE NUTRITIONIST and THE NUTRITIONIST PARTICIPATING IN THE FHP. The results showed the workers from different field of action, expressed the necessity to have the nutritionist to be inserted on the health team and demand to enlarge the field of action of this professional; and that the nutritionist can share its knowledge with the team and the population as a focus of health attention.

KEYWORDS: nutritionist; family health program; work.

RESUMEN. El presente estudio busco analizar el actuar y la importancia del nutricionista en la Estrategia de Salud de la Familia, sobre el ángulo de los diferentes profesionales de salud que actúan en las Unidades Básicas de Salud (UBS) y Unidades de Salud de la Familia (UBSF), de la Municipalidad de Colombo-Brasil. Es una investigación exploradora, cuyo objetivo principal fue evaluar el conocimiento de esos profesionales, referente al papel del Nutricionista en los Programas de Salud de la Familia. Los datos fueron cogidos a través de una entrevista seme-estructurada, aplicada a 12 profesionales de salud. Fue respetada la resolución 196/ 96 que reglamenta la investigación con seres humanos. De los datos obtenidos se construyeron las categorías que siguen: CONOCIENDO AL NUTRICIONISTA y EL NUTRICIONISTA PARTICIPANDO DEL PSF. Los resultados mostraron que los profesionales de las diferentes áreas de la salud, sienten la necesidad, de que el profesional Nutricionista actué y haga parte de los Equipos de Salud, sumando conocimientos con los demás integrantes y principalmente compartirlos con la comunidad, que es ella el centro de la atención de la salud.

PALABRAS-CLAVE: nutricionista; programa salud de la familia; trabajo.

Recebido em: 19/02/2005

Aceito em: 28/07/2005
Anderson Carlos dos Santos

Rua Miguel Jorge Nassa, 991 - Ap. 41 - Bloco 02 - B. Tingüi 82620-140 - Curitiba - PR

E-mail: andio@ig.com.br 


\section{INTRODUÇÃO}

Nas últimas décadas, o Brasil conquistou grandes avanços no campo da saúde, onde deve ser considerado que sua principal conquista foi o processo de construção do Sistema Único de Saúde (SUS), regulamentado pela Constituição Federal de 1988 e pelas Leis Complementares, tendo como principais pilares: universalização, integralidade, descentralização e participação popular.

Porém podemos afirmar que o modelo assistencial ainda predominantemente no Brasil é caracterizado pela prática médica voltada para uma abordagem biológica e intra-hospitalar, associada a uma utilização irracional dos recursos tecnológicos, apresentando, na grande maioria das vezes, cobertura e resolubilidade baixas e de alto custo. Dessa forma, ele gera grande insatisfação nos gestores do sistema, profissionais de saúde e, principalmente nos usuários.

Nesse contexto, o Ministério da Saúde assumiu a partir de 1994, a Estratégia de Saúde da Família (ESF), visando a reorganização do modelo tradicional, por intermédio da reesquematização da atenção à saúde, em conformidade com os princípios do SUS ${ }^{1}$.

Cada equipe do Programa de Saúde da Família (PSF) é composta, no mínimo, por um médico, um enfermeiro, um auxiliar de enfermagem e entre quatro e seis Agentes Comunitários de Saúde (ACS). Outros profissionais, a exemplo de dentistas, assistentes sociais e psicólogos, poderão ser incorporados às equipes ou formar equipes de apoio, de acordo com as necessidades e possibilidades locais, pois a Unidade Básica de Saúde da Família pode atuar com uma ou mais equipes, dependendo da concentração de famílias no território sob sua responsabilidade ${ }^{1}$.

O presente trabalho visou problematizar a ausência do nutricionista na ESF, utilizando o olhar de diferentes trabalhadores de saúde. Nessa perspectiva, pretende apresentar argumentos que possam auxiliar os profissionais de nutrição na discussão da inserção do Nutricionista nesse mercado de trabalho que é o PSF, modelo proposto pelo governo brasileiro para promover, prevenir e recuperar a saúde da população.

\section{REFERENCIAL TEÓRICO}

Dados de recentes pesquisas, demonstram que o perfil de saúde da população brasileira e a transição epidemiológica em curso no Brasil, fruto das mudanças no perfil demográfico e nutricional da população, vêm promovendo profundas alterações no padrão de morbimortalidade e no estado nutricional em todas as faixas etárias, em todo o espaço geográfico, nas áreas urbana e rural das grandes e pequenas cidades ${ }^{2}$.

Pode-se citar a pesquisa da Sociedade Brasileira de Endocrinologia Médica (SBEM) denominada Projeto Escola Saudável que demonstra que para a "população de 6 a 18 anos, existam pelo menos 6,7 milhões de obesos, se mantidas as taxas do último levantamento de 1997" ${ }^{3}$. Entre as crianças também houve aumento da obesidade, pois o número de crianças acima do peso subiu de $3 \%$ para $15 \%$ entre 1975 e 1997. No fim da década de 1990, sabia-se que havia aproximadamente 6,5 milhões de crianças obesas, enquanto entre adultos homens a variação foi de $3 \%$ a $7 \%$; entre as mulheres, $8 \%$ a $13 \%{ }^{3}$.

De outra parte, são inúmeros os problemas inerentes à alimentação e à nutrição inadequadas. Nos países em desenvolvimento, cerca de $55 \%$ das mortes infantis estão ligadas à desnutrição, não existindo na história da humanidade situação mórbida desta magnitude, apesar dos avanços na redução da prevalência do problema ${ }^{4}$. No Brasil, mesmo com a diminuição em mais de $20 \%$ da desnutrição protéica energética (DEP) na última década, um contingente considerável de crianças brasileiras ainda apresentam atraso marcante no crescimento, pela relação peso/ idade, na faixa crítica dos 6 aos 23 meses ${ }^{5}$.

Também se pode constatar um crescimento acentuado na população com idade superior a 60 anos, trazendo consigo o aumento das doenças nãotransmissíveis. Na população adulta detecta-se a elevação das doenças como diabetes, obesidade, neoplasia, hipertensão arterial e hiperlipidemias. Entre os adolescentes a obesidade e hiperlipidemias também preocupam os envolvidos com a saúde pública ${ }^{2}$. 
A Organização Mundial de Saúde recomenda que os países adotem estratégias de prevenção primária para modificar a dieta da população, a fim de alcançar ingestas de nutrientes dentro dos limites aconselhados ${ }^{3}$. As recomendações específicas referem-se à diminuição da ingestão de gorduras, ao aumento de alimentos amiláceos e a uma ingestão considerável de frutas e hortaliças.

Os dados de pesquisa realizada por Mondini e Monteiro apresentaram resultados opostos aos preconizados pela OMS. Segundo eles o consumo de lipídios aumentou no Brasil de $26 \%$ para $29 \%$ e o de frutas caiu de $4 \%$ para $2,5 \%$ no período compreendido entre 1962 e 1968.

O PSF foi criado oficialmente pelo Ministério da Saúde em 1994, a partir do reconhecimento da crise do modelo de atenção à saúde voltado para ações curativas que privilegiavam a medicina de alto custo, verticalizada, excludente e de baixo impacto na melhoria da qualidade de vida da comunidade. Este programa assume, como foco, a reorganização da atenção básica, garantindo a oferta de serviços à população brasileira e também o fortalecimento dos princípios da universalidade, acessibilidade, integralidade e equidade do SUS ${ }^{6}$.

De acordo com pesquisa realizada por Machado sobre o perfil de recursos humanos no PSF em 1999, em todo o Brasil havia 2703 médicos (51,3\%) e 2561 enfermeiros (48,7\%), demonstrando que a composição mínima das equipes vinha sendo cumprida de acordo com a recomendação do Ministério da Saúde. Contudo, ao investigar a participação de outros profissionais de saúde nas equipes de Saúde da Família, verificaram que estes são muito reduzidos, pois o assistente social aparece em 9,3\% das equipes, o psicólogo em $5,3 \%$ e o nutricionista em $4,5 \%{ }^{7}$.

Atualmente, observa-se que, apesar da importância da ação destes profissionais, eles não atuam em todas as UBSF e não estão incluídos nas ESF, o que nos motivou a realizar este estudo.

\section{OBJETIVOS}

Avaliar o conhecimento dos trabalhadores de saúde sobre o papel do nutricionista em Programas de Saúde da Família.

\section{REFERENCIAL METODOLÓGICO}

Para o desenvolvimento deste estudo, foi utilizada a metodologia qualitativa, por favorecer o conhecimento de crenças e valores do indivíduo, permitindo compreender o significado das ações e das relações humanas. Isso proporciona o conhecimento da dinâmica e da estrutura da situação em estudo sob o ponto de vista de quem a vivencia ${ }^{7}$. O modelo exploratório-descritivo foi usado para que pudéssemos descrever a opinião dos trabalhadores de saúde que atuam no PSF, no município de Colombo, PR, sobre a importância da inserção do nutricionista na Estratégia de Saúde da Família.

A amostra foi composta por 12 trabalhadores da saúde: um médico, quatro enfermeiras, dois técnicos de enfermagem, uma auxiliar de enfermagem, três agentes comunitários de saúde, um agente de saúde. Do total da amostra, somente uma enfermeira, um técnico de enfermagem e um agente de saúde não atuavam no PSF, os quais trabalhavam em Unidade Básica de Saúde.

Os dados foram coletados em uma UBSF e UBS do Município de Colombo, Pr, no período de janeiro a março de 2004, por meio de entrevistas semi-estruturadas, que foram gravadas para manter a fidedignidade das informações obtidas.

Respeitou-se o contido na resolução 196/96 que regulamenta pesquisa com seres humanos ${ }^{8}$.

\section{ANÁLISE E DISCUSSÃO DOS DADOS}

Os relatos desses trabalhadores foram discutidos, analisados e agrupados e nos permitiram construir as seguintes categorias e subcategorias: 1) CONHECENDO O NUTRICIONISTA. 2) NUTRICIONISTA PARTICIPANDO DO PSF, que dá origem às seguintes subcategorias: a) o nutricionista inserido na equipe multiprofissional; b) Necessidade da inserção do nutricionista em equipes de saúde; c) motivos da não inserção do nutricionista em equipes de saúde.

Na categoria CONHECENDO O NUTRICIONISTA, observou-se que a maioria dos entrevistados 
desconhece o papel deste profissional, suas funções e atribuições, pois eles referem como função deste profissional apenas a elaboração de dieta. Isto é observado nas falas a seguir:

Ele faz o balanceamento da dieta, ele cuida da dieta do paciente (Técnico de Enfermagem, UBS).

Antes (...), a minha visão era que o nutricionista ajudava no regime para emagrecer (...) sei o quanto é importante, não só para um regime de estética, como também para saúde? As dietas equilibradas, exercícios, eu acho muito importante (...) (Enfermeira, PSF).

Seria aquele profissional que sabe fazer uma dieta equilibrada para cada tipo de paciente conforme as necessidades, conforme o perfil do paciente, na obesidade ou magro, de acordo com o que ele mais necessita (Médico, PSF).

Este fato corrobora o que Bosi afirma, ou seja, "mesmo após trinta anos de reconhecimento legal, o nutricionista continua a ser visto como um calculador de dietas" 9:205.

O mais preocupante é que este profissional ainda é confundido com o médico, demonstrando claramente a centralização no ato médico, como mostra a fala de um dos entrevistados, que afirma que o nutricionista é:

Um médico importante como outro (...) para orientar na alimentação, é uma pessoa importante como outro médico (Agente Comunitário de Saúde).

Verificou-se também que o nutricionista ainda é visto como profissional voltado para o atendimento da classe dominante, demonstrando claramente o antagonismo entre as classes sociais em nosso país.

Antigamente a gente via a nutricionista como um atendimento só para elite (...) (Enfermeira, PSF).

É a primeira vez que vejo nutricionista numa Unidade de Saúde (...) (Técnica de enfermagem, PSF).

Apesar do exposto, o Conselho Nacional de Educação (CNE) define o Nutricionista da seguinte forma:

"Nutricionista, com formação generalista, humanista e crítica, capacitado a atuar, visando à segurança alimentar e à atenção dietética, em todas as áreas do conhecimento em que alimentação e nutrição se apresentem fundamentais para a promoção, manutenção e recuperação da saúde e para a prevenção de doenças de indivíduos ou grupos populacionais, contribuindo para a melhoria da qualidade de vida, pautado em princípios éticos, com reflexão sobre a realidade econômica, política, social e culturais" ${ }^{10}$.

Por isso afirmamos que a concepção de nutrição envolve muitos aspectos, cuja amplitude e interdisciplinaridade não podem ser monopólio de uma única categoria profissional, pois trabalhar na nutrição não significa somente a prescrição e o cálculo de dietas, mas a luta e também a conquista da cidadania.

A nutrição é a base para todos os processos fisiológicos e patológicos, porque nenhum fenômeno orgânico normal ou anormal ocorre sem que haja um componente nutricional envolvido. Este fato mostra a influência que a nutrição tem na promoção, manutenção e recuperação da saúde. Observa-se, neste estudo, que os profissionais da área de saúde necessitam de melhor entendimento do papel do nutricionista, ampliando o seu conhecimento sobre as funções deste profissional, pois saber "lidar com nutrição é lidar com a vida, mas não apenas com vidas alheias, e sim com valores, concepções, percepções, representações de nossa própria alimentação, porque a nossa cotidianidade tende a prevalecer sobre o saber científico" ${ }^{3}$.

\section{A Categoria O NUTRICIONISTA PARTICIPANDO}

DO PSF nos possibilitou construir as seguintes subcategorias: Nutricionista inserido na equipe Multiprofissional, Necessidade da inserção do nutricionista em equipes de saúde e Motivos da não inserção do nutricionista nas equipes de saúde.

Em Nutricionista inserido na equipe Multiprofissional a maioria dos entrevistados, relatou ser muito importante a participação do nutricionista no dia-a-dia da UBS. Eles referem que nas Unidades de Saúde, onde há residentes nutricionistas, percebe- 
se que muitas dúvidas em relação à conduta dos demais profissionais sobre a alimentação da população puderam ser esclarecidas e mais bem entendidas:

O profissional de nutrição, inserido na equipe aqui, participa de tudo. No sentido de que a gente sentiu a evolução de toda equipe, não só os profissionais de nível superior (...) a equipe de auxiliares pede muito apoio da nutricionista (...) (Enfermeira, PSF).

Este fato reafirma a importância do trabalho em equipe multiprofissional, quando na modalidade de trabalho coletivo, se configura a relação recíproca entre as múltiplas intervenções técnicas e a interação dos agentes de diferentes áreas profissionais, dandose a articulação das ações multiprofissionais e de cooperação.

Os participantes deste estudo também referem que, com a inserção do nutricionista na equipe de Saúde da Família, há melhor divisão do trabalho, diminuindo o acúmulo de funções e sobrecarga de atividades dos outros profissionais e com melhora do atendimento do usuário.

Como o nutricionista ainda não tem lugar assegurado em muitas UBS e UBSF, os profissionais de saúde geralmente acabam desempenhando papel de coadjuvante, assumindo função que não Ihes pertence.

Eu acho que melhoraria a divisão de trabalho, até porque, às vezes, você se sobrecarrega de determinadas coisas, além de você não ter o conhecimento mais aprofundado (...) a gente poderia contribuir para a comunidade com o nutricionista, a gente não tem, acaba que a gente mesmo faz (...) então se houvesse o nutricionista, você dividiria melhor o trabalho e seria muito melhor, seria feito melhor o trabalho para esse paciente. Seria muito mais completa a intervenção nesse paciente (Enfermeira, PSF).

Nas UBS, onde os trabalhadores tiveram pouco ou quase nenhum contato com o nutricionista, os entrevistados citaram dificuldades em abordar temas relacionados com alimentação e nutrição:

Eu acho que ele ajudaria muito, principalmente na conduta dos agentes de saúde (...) que o agente de saúde consegue chegar na família, onde, às vezes, outros profissionais não chegam. Então a gente tem muita dúvida para responder às questões que se referem à nutrição. Eu acho que se houvesse nutricionista junto, daria base e suporte à equipe (Agente de Saúde, UBS).

Observou-se também que a falta deste profissional, inserido na equipe, abre lacunas para que outros profissionais se apropriem, de forma inadequada ou superficial, do conhecimento sobre nutrição, tentando suprir esta falta, exercendo as funções deste profissional, sem ter conhecimento aprofundado de alimentação e nutrição. O conhecimento desses profissionais é adquirido ao longo da sua trajetória, desde sua formação profissional, perpassando pela sua atuação nas UBS e também adotando informações obtidas através da mídia.

A gente até orienta as pessoas.

A Sra. Está com a pressão muito alta, quem sabe se a senhora diminuísse o peso ou se mudar a alimentação quem sabe até melhorasse (...).

(...) esses dias eu até vi uma pesquisa no Fantástico sobre a vitamina $C$ (...) (Auxiliar de Enfermagem, PSF).

Na puericultura, o que a gente faz? Pesa a criança, mede, pergunta o que a criança come, a alimentação. Às vezes a gente não dá nenhuma orientação mas na maioria das vezes a gente orienta a mãe sobre os alimentos que deve dar à criança (Enfermeira, PSF).

Os médicos e enfermeiros, que participaram deste estudo, apontam as dificuldades que têm para orientar seus pacientes sobre alimentação. Eles relatam que não têm formação adequada nesta área, pois o currículo de seus cursos não possui disciplina específica de nutrição ou, quando possui, apresenta um conteúdo muito superficial sobre o tema. Vale ressaltar que são esses profissionais que esclarecem a população sobre temas relativos à alimentação, mesmo quando não possuem qualificação adequada para desempenhar essas funções.

A nossa cadeira de nutrição na enfermagem é muito pouco, mas a equipe de atenção básica, auxiliar, técnica e ACS, não tem nada! (Enfermeira, PSF).

Eu, durante o curso de medicina, não vi, não tive nenhuma matéria relacionada à nutrição, nenhuma! (...) 
você aprende lá dieta hipossódica, hipercalórica; mas não estuda o que é uma dieta nesse sentido, você não sabe nada (...) então é uma área em que nós temos deficiência (...) Eu nem sei orientar, essa que é a verdade. Não adianta querer falar, mas o médico não sabe orientar, ele tem uma idéia básica, pouco mais que o paciente (Médico, PSF).

Esses relatos corroboram os resultados obtidos em pesquisas em que foram avaliadas as dificuldades que os médicos e enfermeiros têm na abordagem de problemas alimentares. Os médicos reconhecem que ocorreram deficiências na sua formação e ressentem-se da precariedade e inconsistência dos conhecimentos no campo da nutrição. As informações adquiridas na prática não são sistematizadas; eles não têm visão global do assunto. No caso dos enfermeiros, têm-se resultados semelhantes; eles referem que na graduação tiveram disciplina específica sobre nutrição; mas avaliaram o ensino negativamente, fazendo críticas à metodologia e à falta de aplicação prática dos conhecimentos adquiridos em relação à nutrição ${ }^{11}$.

Todos esses fatos, demonstram que é imprescindível a participação e a inserção do nutricionista em equipes de saúde, porque:

A identificação do problema alimentar requer do profissional conhecimentos sólidos de nutrição e dietética. Pressupõe-se que os profissionais, cujo trabalho tem relação com a promoção da saúde, prevenção de doenças ou recuperação da saúde deva não só conhecer os processos nutricionais, mas estar preparado para aquilatar a influência dos fatores nutricionais nos problemas que se apresentam na prática profissional, atribuindo a eles a devida importância ${ }^{11}$.

Essa deficiência em relação às questões alimentares também são encontradas nas falas dos técnicos e auxiliares de enfermagem e dos ACS. Eles referem que nem eles, nem os médicos estão preparados para lidar com as questões alimentares que aparecem em seu cotidiano.

Mesmo para orientar a gente, porque tem muita coisa que a gente fica em dúvida (Auxiliar de Enfermagem, PSF).
Por exemplo, a gente tem uma família que tem crianças desnutridas, o médico não vai saber exatamente o que fazer, já o nutricionista sabe, porque ele estudou para isso. O médico muitas vezes tem alguma dúvida que o nutricionista pode auxiliar (ACS).

Na subcategoria, Necessidade da Inserção do Nutricionista em Equipes de Saúde, os participantes, deste estudo, ressaltaram a importância deste profissional estar atuando nas equipes de atenção primária e citam as dificuldades encontradas quando, os mesmos se vêem "obrigados" a exercer o papel de nutricionista que não lhes cabe:

Eu não sou nutricionista.Como eu vou dar uma orientação se eu não sou nutricionista? (Enfermeira, UBS).

Não é área da gente, a gente sabe por alto, não pode comer isso, não pode comer aquilo, mas é o nutricionista vai dar orientação correta, não é mesmo? (Auxiliar de Enfermagem, PSF).

O enfermeiro e o médico não têm essa formação para essa área alimentar. Eles dão aquela orientação mais por cima, mais superficial; nenhum tem o conhecimento que o nutricionista tem (Enfermeira, PSF).

De acordo com relatório produzido na $11^{\mathrm{a}}$ Conferência Nacional de Saúde ${ }^{13}$, observou-se a importância da participação de outros profissionais de saúde, incluindo a do nutricionista, nas equipes de Saúde da Família, proporcionando um atendimento mais qualificado e dentro do princípio da integralidade da atenção:

(...) no caso de opção por essas estratégias como forma de organização, deverá ser garantida a sua adequação às necessidades e prioridades locais, sem modelos programáticos verticalizados, garantindo a atuação de outros profissionais de saúde como psicólogos, nutricionistas, assistentes sociais, fisioterapeutas, fonoaudiólogos e outros, proporcionando uma atenção multiprofissional de acordo com a realidade de cada município (...) ${ }^{13}$.

Essa necessidade também foi expressa pelos profissionais que compuseram a amostra deste estudo, conforme a fala a seguir: 
Eu acho que um complementa o outro. Médicos, nutricionistas, enfermagem (...) acho que se não tiver um nutricionista, sempre vai estar faltando alguma coisa (...) (Enfermeira).

A participação do nutricionista e sua integração nas equipes do PSF está estabelecida em sua formação acadêmica, que o instrumentaliza a realizar diagnóstico nutricional da população, tornando-o, assim, o único profissional a receber um conhecimento específico que lhe permite, a partir do diagnóstico e da observação dos valores socioculturais, propor as devidas orientações dietéticas, adequando-as à realidade de cada unidade familiar; portanto um profissional apto a colaborar para reafirmar o modelo de atenção à saúde proposto em nosso país ${ }^{2}$.

Nas UBS e também nas UBSF, muitas vezes, é priorizada a medicalização, ao invés da prevenção, pois os profissionais afirmam não estarem preparados para resolver questões sobre alimentação dando ênfase às atividades desempenhadas pelo nutricionista.

Às vezes o pessoal acha que não precisa, mas o nutricionista a gente vê a doutora trabalhando aqui, quanto é importante, quantas pessoas são orientadas nas visitas, coisa que nós, médicos, não nos ligamos de orientar. Às vezes não temos a paciência nem o conhecimento de certos alimentos, que o nutricionista vai orientar e vai dar saída para o caso. Nós nos preocupamos em medicar aquilo que já está doente, que é um erro, em vez de nós prevenirmos com dietas, com alimentação, coisas que vão prevenir doenças que amanhã depois, o próprio estado, o próprio governo, está gastando para tratar duma coisa que foi gerada em função de uma desnutrição ou de uma dieta mal orientada (...) (Médico, PSF).

A dificuldade que os profissionais médicos e enfermeiros encontram para lidar com as questões de alimentação decorrem da complexidade do problema em si, do seu desconhecimento sobre ele e dos conflitos que emergem das contradições entre o que se sabe e o que se pensa, em relação direta com a atividade que se faz na prática. Essa situação acaba gerando uma não valorização dos problemas alimentares e nutricionais, impedindo um trabalho em equipe ${ }^{3}$.
Na Subcategoria motivos da não inserção do nutricionista nas equipes, os entrevistados referemse à vontade política dos gestores de saúde em relação à abertura de campo de trabalho a este profissional. Eles acreditam que para isto ocorrer, deve haver mudança da mentalidade dos gestores, vontade política e conhecimento da realidade vivida pela população.

Eu acho que precisa é uma mudança de mentalidade, mudança de mentalidade do sistema que está operando (...) (Médico, PSF).

É uma vontade política e tem muito que ver com verba mesmo. Tem espaço e tem necessidade, mas os políticos não têm essa visão e é um caminho longo a ser percorrido (Enfermeira, PSF).

Eu acho que se tinha de colocar isso para os secretários de saúde, para o povo lá em cima, mostrando a importância e a realidade da população que vive aqui (Auxiliar de Enfermagem, PSF).

A inserção dom nutricionista nas equipes de PSF é necessária e está atrelada à visão e vontade política.

Também foi citado nas entrevistas que o nutricionista ainda não é valorizado em face aos outros profissionais de saúde:

(...) hoje o nutricionista não é valorizado, porque o pessoal não aprendeu a fazer a medicina profilática, de cuidar do paciente antes dele adoecer (...) Então esse profissional não é valorizado, porque os próprios governantes, os próprios coordenadores, os próprios secretários de saúde, os próprios profissionais da área como os próprios médico e, enfermeiros desconhecem o potencial que o nutricionista pode dar para à sociedade (...) e o médico sozinho tem praticado uma medicina deficitária; por isso as doenças estão aí e nunca é resolvido, porque falta uma visão total do ser humano e o nutricionista entra para orientar nesse sentido (Médico, PSF).

Esses resultados estão de acordo com os resultados obtidos por Boog ${ }^{11}$ em sua pesquisa, em que os profissionais de saúde entrevistados citam uma visão técnica, que corresponde à conscientização dos profissionais de saúde sobre os problemas de nutrição, para que estes venham futuramente a 
reconhecer o papel do nutricionista, bem como na eficácia de experiências como a Residência Multiprofissional em Saúde da Família. Neste modelo os residentes nutricionistas, têm oportunidade de atuar em equipes de PSF, divulgar suas ações e ampliar o campo de trabalho do nutricionista.

\section{CONSIDERAÇÕES FINAIS}

A partir dos elementos apresentados, percebe-se, de forma clara, que atualmente prevenção, controle e tratamento dos agravos à saúde estão relacionados direta ou indiretamente com a alimentação dos indivíduos.

Segundo as prerrogativas do Ministério da Saúde, o PSF constitui estratégia que prioriza as ações de promoção, proteção e recuperação da saúde dos indivíduos e da família de forma integral e contínua. Para que essa nova prática se concretize, faz-se necessária a presença de um profissional com visão integral do indivíduo, família e comunidade, capaz de atuar de forma humanizada, competente e resolutiva, atendendo às necessidades da comunidade ${ }^{13}$.

A competência do nutricionista para integrar as Equipes de Saúde da Família está contemplada em sua formação acadêmica, o que lhe proporciona conhecimentos que o torna capaz de gerar impactos positivos no perfil epidemiológico da população. Tratase,portanto, de um profissional apto a participar efetivamente da recriação das práticas de atenção à saúde no Brasil.

Essa afirmação fica evidente nas entrevistas realizadas junto aos trabalhadores de saúde do município de Colombo, PR.

Apesar disso, observou-se neste estudo o seguinte elenco escalonado:

a) A maioria dos Trabalhadores de saúde que compuseram a amostra desconhecem a real função do nutricionista e suas atribuições, associando o conceito deste profissional a prescrição de dietas, cuja atuação está voltada para o atendimento das classes dominantes, demonstrando o antagonismo entre as classes sociais em nosso país. É importante que os profissionais de saúde possuam melhor preparo e entendimento sobre o papel do nutricionista, para que haja melhor conscientização da importância da sua inserção nas equipes de saúde.

b) Nas Unidades de Saúde da Família onde há residentes nutricionistas, houve mudanças no comportamento destes profissionais, em relação às questões e condutas referentes à nutrição dos indivíduos e famílias atendidos pelo programa, o que denota um ponto positivo que reforça a possibilidade futura de que esses profissionais se insiram nas Equipes de Saúde da Família.

c) A falta do profissional nutricionista nas Equipes de Saúde da Família abre espaço a outros profissionais de saúde para que se apropriem, de forma superficial, do conhecimento sobre alimentação e nutrição, apesar de mesmos estarem conscientes de que não possuem formação adequada na área de nutrição, já que, no currículo dos seus cursos, não existe disciplina relativa à área de nutrição; ou, quando possui, apresenta conteúdo muito superficial do tema. Vale ressaltar que serão esses profissionais que estarão abordando temas relativos a alimentação, quando não houver o profissional qualificado para desempenhar essas funções.

d) A ausência ou a pouca inserção do nutricionista no PSF, na visão dos trabalhadores de saúde, está intimamente ligada a vontade política dos gestores públicos que ainda não se sensibilizaram da importância da atuação deste profissional. Atualmente sabe-se que a abordagem alimentar e nutricional ganha relevância e está associada à melhoria do perfil epidemiológico da população, ocasionando a diminuição dos gastos com saúde pelo Estado, o que nos indica a notória necessidade de ampliar a prestação de assistência alimentar e nutricional à população. 
A tentativa de entrevistar vários tipos de trabalhadores de saúde, das mais diferentes áreas de atuação, obtendo sua visão do profissional nutricionista, buscou explicitar que este profissional é imprescindível, quando o assunto é alimentação e nutrição. Isso ficou bem claro desde a primeira entrevista, demonstrando que os próprios trabalhadores da área de saúde sentem a necessidade e reivindicam que o campo de atuação deste profissional seja ampliado e que o mesmo possa vir a somar seus conhecimentos técnicos com os demais membros da equipe de saúde e principalmente repasse à sociedade e à população que deve ser o centro de toda a atenção à saúde.

Se nós, como categoria profissional, temos a convicção de que, de acordo com a nossa formação, temos um instrumental teórico prático e que podemos, por meio dos nossos esforços, exercer nossa função perante às necessidades e direitos nas questões de saúde da população, devemos lutar de forma organizada, unindo esforços de toda categoria e entidades profissionais, para que o nutricionista seja inserido em todas as políticas públicas de saúde implantadas em âmbito nacional, estadual e municipal, sem que para isso seja necessária a força de legislações.

\section{REFERÊNCIAS}

1 Almeida M, organizador. Diretrizes curriculares nacionais para os cursos universitários da área da saúde. Londrina: Rede Unida; 2003. p.37-44.
2 Assis AMO, Santos SMC, Freitas MCS, et al. O Programa Saúde da Família: contribuições para uma reflexão sobre a inserção do nutricionista na equipe multidisciplinar. Rev Nutr. PUCCAMP 2002; 15(3):255-66.

3 Sociedade Brasileira de Endocrinologia. Projeto Escola Saudável. Disponível em: <http://www.endocrino.org.br/ com_noticias.htm\#18 > (21 oct 2003).

4 Fundo de Nações Unidas Para a Infância. Situação mundial da infância; Brasília; 1998.

5 Ministério da Saúde(BR).Secretaria de Assistência à saúde. Política Nacional de Alimentação e Nutrição. Brasília (DF); 2003. p.48.

6 Boog MCF. Dificuldades encontradas por Médicos e Enfermeiros na abordagem de problemas alimentares. Rev Nutr. PUCCAMP 1999; 12(3):261-72.

7 Minayo MCS. O desafio do conhecimento: pesquisa qualitativa em saúde. Rio de Janeiro - São Paulo, Abrasco - Hucitec; 1992.

8 Ministério da Saúde (BR). Conselho Nacional de Saúde. Resolução no 196 de 10 de outubro/1996. Diretrizes e normas regulamentadoras de pesquisa envolvendo Seres Humanos: Brasília (DF); 1997.

9 Bosi MLM. Profissionalização e conhecimento: a nutrição em questão. São Paulo: Hucitec; 2000. p. 205.

10 Conselho Nacional de Educação (BR). Resolução CNE/ CES n 5 de 07 de novembro de 2001.

11 Boog MCF. Educação nutricional em serviços públicos de saúde. Cad Saúde Publ; 1999; 15 (supl 3):139-47.

12 Peduzzi M. Equipe multiprofissional de saúde: conceito e tipologia. Rev Saúde Publ 2001; 35(1):103-9.

13 Ministério da Saúde (BR). $11^{\text {a }}$ Conferência Nacional de Saúde, Brasília 15 a 19 de dezembro de 2000: O Brasil falando como quer ser tratado: efetivando o SUS: acesso, qualidade e humanização na atenção à saúde com controle social: Relatório final / Ministério da Saúde, Conselho Nacional de Saúde.Brasília; 2003.

14 Secretaria de Políticas de Saúde (BR). Departamento de Atenção Básica. Programa Saúde da Família. Rev Saúde Publ 2000; 34(3):316-9. 